\title{
Human Epidermal Growth Factor Receptor-2(HER-2) expression in Sri Lankan gastric adenocarcinoma patients: An analysis of the concordance of HER2 expression and survival based on silver in-situ hybridization (SISH)
}

Duminda Subasinghe ( $\square$ dumindas1982.hpb@gmail.com )

Department of Surgery,University of Colombo

Nathan Acott

PathWest Laboratory Medical WA: PathWest Laboratory Medicine Western Australia

PKB Mahesh

PGIM: Postgraduate Institute of Medicine University of Colombo

Sivasuriya Sivaganesh

Department of Surgery, University of Colombo

Ananthi Samarasekera

Department of Pathology,The National Hospital of Sri Lanka

Marian Priyanthi Kumarasinghe

PathWest Laboratory Medical WA: PathWest Laboratory Medicine Western Australia

Dharmabandu Nandadeva Samarasekera

Department of Surgery, University of Colombo

Menaka Dilani Samarwickrema Lokuhetty

Department of Pathology,University of Colombo

\section{Research Article}

Keywords: Gastric carcinoma, HER2, SISH, IHC, Survival, Concordance

Posted Date: March 17th, 2021

DOI: https://doi.org/10.21203/rs.3.rs-298497/v1

License: (우 (i) This work is licensed under a Creative Commons Attribution 4.0 International License. Read Full License 


\section{Abstract}

Background: Accurate HER2 status is crucial for gastric adenocarcinoma (GC) patient selection for antiHER2 therapy. It is assessed immunohistochemically (IHC) for protein expression, and by silver in-situ hybridization (SISH) for gene copy number. This study aimed to evaluate the concordance of HER2 status by IHC/SISH analyses and HER2-SISH based survival.

Methods: This prospective study includes 145 GC's (excluding gastro-oesophageal-junction tumours) from the National Hospital of Sri Lanka with defined demographic, clinical-radiological-pathological characteristics. HER2IHC was assessed by DAKO A0485, RealTM Envision system and interpreted using Ruschoff criteria. HER2-SISH was assessed with INFORM HER2 dual ISH DNA Probe Cocktail. Concordance between HER2 IHC/SISH results was determined by Cohens kappa statistics. The association between the survival and HER2-SISH positivity was evaluated using the cox-regression method. Adjustments were done for age, gender, Lauren classification, tumour location and the tumour staging.

Results: Of the 69 gastrectomies and 76 biopsies, 8.3\% $(n=12)$ were HER2-IHC positive $(n=7,+2$ and $n=5,+3)$. HER2-SISH positivity was $4.8 \%(n=7)$. All IHC +3 were SISH positive, while two +2 cases were SISH positive. Concordance for IHC $0,+1,+3$ were $100 \%$. There was a significant overall correlation (kappa $=0.72, p<0.001$ ) between HER2-IHC and HER2-SISH indicating substantial concordance. The mean overall survival of HER2-SISH negative and positive patients were $41.7(0-210)$ and $14.6(3-51)$ weeks respectively, after a mean duration of patient follow up for 40.4 weeks (range $0-210)$. Survival was relatively lower $(p=0.001)$ in the group with HER2SISH positivity.

Conclusion: HER2-IHC was well concordant with HER2-SISH for $0,+1,+3$ scores and could be used for treatment and prognostication in low resource settings, where SISH facility is unavailable. HER2-IHC + 2, without gene amplification may be due to transcriptional activation by other genes or post-transcriptional events, mandating further evaluation by SISH. Survival of GC patients is significantly affected by HER2-SISH positive status.

\section{Introduction}

HER2 positive gastric carcinomas (GC) are usually associated with more aggressive biological behavior ${ }^{1-6}$. The Trastuzumab for Gastric Cancer (ToGA) trial revealed trastuzumab in combination with chemotherapy, significantly improves the overall survival of patients with HER2 positive advanced GC or gastroesophageal junction carcinoma (GOJC) ${ }^{6}$. Based on the results of the ToGA study in 2010, the Food and Drug Administration (FDA) ${ }^{7}$ in USA and Therapeutic Goods Administration (TGA) in Australia granted approval ${ }^{8}$ for trastuzumab in combination with chemotherapy for the treatment of patients with GC and GOJC. Subsequently this therapy has been approved globally for GC and GOJC ${ }^{8}$.

The reported frequency of HER2 protein overexpression by immunohistochemistry (IHC) in GC ranges from $8.2 \%$ to $40.3 \%^{1-6,9-12}$. Only a single documentation is available from Sri Lanka of HER2 expression by IHC in Sri Lankan GC patients. 12

HER2 status is determined by immunohistochemistry (IHC) and in-situ hybridization (ISH) techniques. IHC is semi-quantitative, evaluating HER2 protein expression. IHC has the advantage of being relatively inexpensive and is widely used in clinical laboratories on formalin-fixed paraffin-embedded tissue sections (FFPE). In contrast, in- 
situ hybridization (ISH) is quantitative, evaluating the HER2 gene copy number. Three types of ISH methods are used for HER2 status determination; fluorescence in situ hybridization (FISH), chromogenic in situ hybridization $(\mathrm{CISH})$ and silver in situ hybridization (SISH) ${ }^{13,14}$. SISH is the preferred method among these different techniques ${ }^{15}$. HER2- SISH status data of Sri Lankan GC patients is unavailable, with paucity of similar data from South Asia.

Sri Lanka has a low incidence of GC in comparison to global and regional countries, with an incidence of 1.2 per 100,000 population and an age adjusted mortality rate of $6.7^{16}$. Current study examined the HER2 status of GC patients in Sri Lanka by IHC and SISH techniques and sought to determine the concordance rate. It also aimed to evaluate HER2-SISH based survival of GC patients who did not receive anti HER2 therapy.

\section{Methodology}

Study setting and ethics: This prospective, collaborative study was conducted at the Departments of Surgery and Pathology, Faculty of Medicine, University of Colombo, Department of Pathology, National Hospital of Sri Lanka (NHSL) and the Department of Anatomical Pathology, Pathwest QE II Medical Centre, Perth, Australia. Ethical approvals were obtained from the ethics committees of the Faculty of Medicine, University of Colombo and the NHSL.

Study population: One hundred and forty-five (145) consecutive GC patients (excluding GOJC cases) presenting to the NHSL over four years (2012 April - 2016 April) were studied and followed up until 2017 December. None of these patients received anti HER2 therapy. HER2 expression by IHC, clinicopathological features and survival finding of the initial 100 patients in the cohort have been documented previously ${ }^{12}$.

In all patients the diagnosis was confirmed by upper gastrointestinal endoscopy (UGIE) and biopsy. Only the gastric resection specimen was included in patients who underwent curative surgery following biopsy. The endoscopic biopsy was included in patients with advanced tumours, who did not undergo gastric resection. Age at diagnosis, gender, type of specimen, tumour location (proximal/distal stomach) and radiological stage assessed by contrast enhanced computerized tomography (CECT) of the abdomen and thorax were documented using a structured data sheet. Radiological data was used to determine the T (tumour), N (nodal with enlargement $>1 \mathrm{~cm}$ ) and $\mathrm{M}$ (metastasis) stages of patients who only had biopsies without resections. Pathological data were used to determine the $\mathrm{T}$ and $\mathrm{N}$ stages in patients who underwent resections. The TNM stage was determined in accordance with the seventh edition of the UICC guidelines ${ }^{17}$.

Pathology: FFPE tumour samples were stained with haematoxylin and eosine. Lauren's classification for GC was used for histological typing (diffuse, intestinal or mixed) ${ }^{18}$.

Immunohistochemistry: FFPE tumour tissue sections were stained for HER2 protein expression using polyclonal rabbit anti-human c-erB-2 oncoprotein (Dako A0485) and Dako Real TM Envision systems. Breast cancer tissue with a HER2-IHC +3 score was used as the positive control. According to Ruschoff scoring [19] a score of $\mathrm{IHC} 0$ or +1 was considered negative for HER2 overexpression, whereas a score of $\mathrm{IHC}+3$ was considered strongly positive. A score of IHC +2 was also considered positive for HER2 overexpression based on criteria by Ruschoff et al.

Silver in situ hybridization: Tissue microarrays (TMAs) were prepared at the Department of Anatomical Pathology, Pathwest QE II Medical Centre, Perth, Australia, using two tissue cores with a diameter of $0.6 \mathrm{~mm}$ extracted from 
each tumor using the TMA arrayer (TMA Master 1.16 SP1). Each TMA also contained various non-gastric tissue samples as controls. Sections of $4 \mu \mathrm{m}$ obtained from the TMA blocks were used for SISH/IHC. The slides were stained using automatic staining devices: the Benchmark Ultraview (Ventana Medical Systems).

The INFORM HER2 dual ISH DNA Probe Cocktail was used for HER2 SISH as the probe. This was designed to quantitatively detect amplification by light microscopy of the HER2 gene and the centromere portion of chromosome 17(CEP17), via two colour chromogenic in situ hybridization(ISH) in FFPE GC tissue, following staining on Ventana automated slide strainers. During SISH signal counting, a discrete signal was counted as a single copy of HER2 or CEP17. HER2 SISH signals (black) are typically smaller in size and more discrete in appearance than CEP17 SISH signals (red), due to differences in target sizes and detection chemistries. During the signal interpretation process 20 cells were counted for red (CEP 17-Chromosome 17 centromere) and black (HER2 signals). HER2 gene status was classified as non-amplified (HER2/Chr17 ratio < 2.0) or amplified (HER2/Chr17 ratio $\geq 2.0$ ).

Statistics: The statistical software program SPSS 21(SPSS Inc., Chicago, IL, USA) was used for data analysis.

Evaluation of HER2-IHC vs HER2-SISH: The concordance between HER2-IHC and HER2-SISH was determined by using Cohens kappa statistics.

\section{Survival analysis:}

Initially survival analysis was performed by the Kaplan-Meier method with log-rank test comparing the survival of HER2-SISH positive and negative groups. Subsequently the association between the survival time and HER2-SISH positivity was evaluated using the cox-regression method. Adjustments were done for age, gender, Lauren classification, tumour location and tumour staging.

\section{Results}

Demographic, clinical-radiological-pathological characteristics: There were 69 (47.6\%) gastric resections and 76 (52.4\%) endoscopic biopsies. A male predominance was observed with a male: female ratio of 1.6:1. The mean age at diagnosis was 60.06 (range $32-82$ years). Most tumours ( $n=87,60 \%)$ were located in the proximal stomach and over $60 \%$ of both proximal and distal GC presented at an advanced radiological stage (stage III/IV). Resected gastric specimens were primarily of stage II $(n=35,50.7 \%)$ on pathological staging. The majority $[72(49.7 \%)]$ were of Lauren's intestinal subtype. Again, majority tumours were moderately differentiated $(n=56,38.6 \%)$, while well differentiated, poor differentiated tumours accounted for 38 (26.2\%), and 51 (35.2\%) respectively. (Figures 1,2).

There were $8.3 \%(n=12)$ HER2-IHC positive cases (Score $+2, n=7$, Score $+3 n=5)$ HER2-IHC was negative $(0,+1)$ in the majority ( $n=133,91.7 \%)$ (Figures 3,4$)$.

\section{HER2 by SISH:}

Table 01 shows the demographic, clinical-radiological-pathological characteristics of HER2- SISH positive and negative GC.

HER2-SISH was 4.8\% ( $n=7)$. All HER2-IHC +3 $(n=5)$ cases were SISH positive. Out of the 7, HER2-IHC +2 cases, $\mathrm{SISH}$ positivity was observed in 2 cases. All IHC negative cases $(0$ and +1$)(n=133)$ were confirmed as SISH 
negative (Figures 3-5).

Table 2 depicts the concordance between HER2-IHC positivity and HER2-SISH positivity. The reliability of HER2 assessment using IHC and SISH was analyzed using Cohens-kappa statistics. The kappa value was 0.72 ( $p<0.001)$, indicating a substantial correlation between HER2 status assessment by IHC and SISH.

HER2-SISH expression and survival: The mean duration of patient follow up (89\% patients for five years or until death as the end point) was 40.4 weeks (range 0-210). All have been treated with standard adjuvant chemotherapy with none receiving anti HER2 (Tratuzumab) therapy. The mean overall survival of HER2-SISH negative and positive patients were 41.7 (0-210) weeks and 14.6 (3-51) weeks respectively. The significantly poor overall survival $(p=0.018)$ of HER2-SISH positive patients is illustrated in Figure 6.

SISH-positivity and advanced age were observed to be independently having a higher hazard ratio for mortality as shown in Table 3.

\section{Discussion}

GC accounts for about $10 \%$ of cancer related deaths worldwide with a case fatality rate of $70 \%$ [20]. Most (70\%) GCs occur in developing countries with half the world's total cases occurring in East Asia ${ }^{21,22}$. The majority are diagnosed at an advanced stage. Despite multiple therapeutic strategies including radical surgery combined with chemotherapy, improving the outcome of advanced stage GC remains a challenge. In recent years, HER2 receptor status has been identified as a novel molecular targeted therapy for GC ${ }^{6}$. Molecular target therapy for GC depends on the accurate evaluation of the HER2 receptor status or the target gene. Therefore, accurate determination of HER2 status is important for molecular targeted therapy.

HER2 positivity by IHC was $8.2 \%(n=12)$, in the study. HER2-IHC has been documented as $9 \%$ in the first 100 patients of this patient cohort (12). IHC $+2,+3$ were considered as positive in both these studies. While HER2-IHC positivity rates in GC have generally ranged from $8.2 \%$ to $40.3 \%$ 1-10 1-6, 9-12, Asian studies have reported rates ranging from $11.7 \%$ to $15.74 \% 23-25$. The explanation for this variation is likely to be multifactorial including differences in the populations studied, use of non-standardized assays using different antibodies and the application of different scoring criteria for interpretation. GOJC's are also reported to have higher HER2 expression rates ${ }^{26}$. The GOJC's were excluded from the current study as these are now considered to be a distinct entity ${ }^{27}$ Exclusion of GOJC and the use of a polyclonal antibody may have contributed to the lower HER2 positivity rate the study encountered. Alternatively, the low HER2-IHC positivity rate could be a reflection of a genuine difference in tumour biology of the local population or due to heterogeneity of HER2 receptor expression which is a well-recognized phenomenon in $\mathrm{GC}^{28}$.

HER2 positivity by SISH was 4.8\% ( $n=7)$ in this study. Four out of 69 resections and 3 out of 76 biopsies showed HER2-SISH positivity. HER2-SISH positivity was more in gastric resections 5.8\% (4/69) whereas only 3.9\% (3/76) cases of biopsy specimens showed positivity. Lower rates of HER2-SISH positivity in biopsies again may have been due to heterogeneity of the HER2 gene amplification ${ }^{29}$.

Most comprehensive data on the concordance between HER2 status by IHC and SISH methods comes from the systematic review and meta-analysis by Pyo et al ${ }^{30}$, which included a total of 12,679 cases from 45 individual studies. Very high concordance rates were found between $0 / 1+$ and $3+$ as was expected. Concordance between 
HER2 IHC2+ and HER2 gene amplification was more variable. The pooled sensitivity and specificity of IHC positivity (when both 2+ and 3+ were considered positive), in predicting ISH confirmed HER2 positivity, were 0.86 and 0.91, respectively. In the current study all cases underwent HER2 amplification testing by SISH. 5 of the 12 $\mathrm{IHC}+$ cases were SISH negative; All $5 \mathrm{SISH}$ negative cases were IHC +2. HER2 gene was amplified in all cases with overexpression of the HER2 protein at +3 level, and HER2 gene was not amplified at 0/+1 level, which is consistent with the reported data ${ }^{31}$. Therefore in this cohort of Sri Lankan GC patients HER2-IHC was well concordant with HER2-SISH for $0,+1,+3$ scores. These findings are valuable as HER2-IHC could provide useful information in limited resource settings for treatment and prognostication for patients with HER2 scores of $0,+1$, +3. This is in keeping with the current recommendation that all patients with GC should have their tumours tested for HER2 status at the time of the initial diagnosis with IHC as the first screening method. Those cases with results considered equivocal for HER2 overexpression (2+) should be referred for HER2 analysis by in situ hybridization ${ }^{14}$. This is endorsed by the study findings where, of the $7 \mathrm{IHC}+2$ cases, SISH positivity was observed in only 2 cases.

The subset of cases showing IHC/SISH discordant results could also be due to heterogeneous HER2 protein expression. Chromosomal instability is probably one of the major causes for this heterogeneity. Kameda et al detected HER2 overexpression without amplification and considered that this may indicate that gene amplification may not be the primary mechanism by which the HER2 protein is over expressed in GC ${ }^{32}$. HER2 overexpression may occur by a number of different mechanisms, including transcriptional activation by other genes or post-transcriptional events ${ }^{33}$ Therefore, HER2-IHC 2+ cases represent a subset of patients with uncertain diagnostic characteristics in terms of HER2 status. IHC 2+ cases should be interpreted carefully and necessary confirmatory tests for gene amplification should be carried out.

Many studies have examined the hypothesis that HER2 status could be a predictor of the survival rate in patients with GC. Andreas et al $^{34}$ have shown that there is a significant association between the level of HER2 expression and faster achievement of cancer-free and overall survival, without adjusting for the tumour stage. Brien et al 35 have shown that the pathological stage and HER2 gene amplification are independent prognostic factors of survival in multivariate analysis. According to the ToGA study ${ }^{6}$ median overall survival was 13.8 months in HER2 positive GC treated with trastuzumab compared to 11.1 months in the chemotherapy only arm. In keeping with these findings, HER2-SISH positivity and advancing age were associated with a significantly low overall survival in the present study independently of tumour stage and other confounding factors.

In conclusion, HER2 prevalence by IHC and SISH were $8.2 \%$ and $4.8 \%$ respectively in this Sri Lankan GC patient cohort. The reliability between HER2-IHC and HER2-SISH is satisfactory with substantial agreement. In view of this, HER2 by IHC could be a satisfactory alternative for GC with $0,+1$ and +3 scores, in settings where HER 2 by $\mathrm{SISH}$ is unavailable. Patients with HER2-IHC +2 scores however require confirmatory testing for gene amplification. Additionally, SISH HER2 positivity is associated with a low overall survival among Sri Lankan GC patients who have not received anti HER2 therapy, independently of the tumour stage.

\section{Abbreviations}

(HER-2)-Human Epidermal Growth Factor Receptor-2

GC - Gastric adenocarcinoma 
GOJC-Gastro-oesophageal junction cancer

IHC-Immunohistochemistry

SISH-Silver In Situ Hybridization

CEP 17-centromere portion of chromosome 17

FFPE- Formalin-fixed paraffın-embedded tissue sections

Fluorescence in situ hybridization (FISH),

Chromogenic in situ hybridization (CISH)

\section{Declarations}

\section{Acknowledgments}

Authors wish to acknowledge the National research council research grant(11-100) for funding the research study and the National Science Foundation travel grant (OSTP/2016/03). Dr Medhavini Dissanayake, Dr Sameera Ravishan, Research assistants, Department of Pathology, University of Colombo, are acknowledged for their assistance in data collection and Mrs Kokila Wijesinghe, Senior technical officer Department of Pathology, University of Colombo for guidance on IHC staining. Authors also like to acknowledge Mr. Sithum Munasinghe of Translational Health Research Institute, Western Sydney University for his input on statistical analysis.

\section{Author's contribution}

DS, MDSL, SS, MPK, DNS,AS conceptualized and DS,MDSL, SS designed the work. DS acquired, analyzed and interpreted the data, and wrote the manuscript. MPK and NA helped with Immunohistochemistry work up. MPK helped with supervision on immunohistochemistry interpretation. PKBM helped with Statistical analysis manuscript drafting. All authors read and approved the final manuscript.

Funding-This study was funded by National Research Council, Sri Lanka by providing the research grant (11-100). Overseas phase of this study was funded by National Science Foundation for providing me a travel grant (OSTP/2016/03).

Availability of Data - The datasets used and analyzed during the current study are available from the corresponding author on reasonable request.

\section{Ethics approval and consent to participate}

This study has been approved by the Ethics review committee of the Faculty of Medicine, University of Colombo (EC 11-139) and the Ethics Review committee of the NHSL. All patients gave their written informed consent to collection and use of their data for research purposes. No individual patients can be identified by the anonymous data used in this study.

Consent for publication - Not applicable. 
Competing interests -The authors declare that they have no competing interests

\section{References}

1. Kim KC, Koh YW, Chang HM, Kim TH, Yook JH, Kim BS,Jang SJ, Park YS. Evaluation of HER2 protein expression in gastric carcinomas: comparative analysis of 1,414 cases of whole-tissue sections and 595 cases of tissue microarrays. Ann Surg Oncol 2011; 18: 2833-2840

2. Baykara M, Benekli M, Ekinci O, Irkkan SC, Karaca H, Demirci U, Akinci MB, Unal OU, Dane F, Turkoz FP, et al. Clinical Significance of HER2 Overexpression in Gastric and Gastroesophageal Junction Cancers. J Gastrointest Surg 2015;19:1565-1571

3. Jørgensen JT. Targeted HER2 treatment in advanced gastric cancer. Oncology 2010; 78: 26-33

4. Kurokawa Y, Matsuura N, Kimura Y, Adachi S, Fujita J, Imamura H, Kobayashi K, Yokoyama Y, Shaker MN, Takiguchi S, Mori M'Doki Y. Multicenter large-scale study of prognostic impact of HER2 expression in patients with resectable gastric cancer. Gastric Cancer 2015, 18(4):691-7.

5. Park DI, Yun JW, Park JH, Oh SJ, Kim HJ, Cho YK, Sohn Cl, Jeon WK, Kim BI, Yoo CH, Son BH, Cho EY, Chae SW,Kim EJ, Sohn JH, Ryu SH, Sepulveda AR. HER-2/neu amplification is an independent prognostic factor in gastric cancer. Dig Dis Sci 2006; 51: 1371-1379

6. Bang YJ, Van Cutsem E, Feyereislova A, Chung HC, Shen L,Sawaki A, Lordick F, Ohtsu A, Omuro Y, Satoh T, Aprile G,Kulikov E, Hill J, Lehle M, Rüschoff J, Kang YK. Trastuzumab in combination with chemotherapy versus chemotherapy alone for treatment of HER2-positive advanced gastric or gastro-oesophageal junction cancer (ToGA): a phase 3, openlabel, randomised controlled trial. Lancet 2010; 376: 687-697

7. FDA Approves Herceptin For HER2-Positive Metastatic Stomach Cancer .First Targeted Medicine Shown to Improve Overall Survival in HER2-Positive Stomach and Gastroesophageal Junction Cancers. https://www.gene.com/media/press-releases/13007/2010-10-20/fda-approves-herceptin-for-her2-positive

8. Therapeutic Good administration. Australian Public Assessment Report for Trastuzumab (Internet). Canberra: Australian Government Department of Health; 2015 [cited 2019 Feb 25). Available from :https://www.tga.gov.au/sites/default/files/auspar-herceptin.pdf

9. Takehana T, Kunitomo K, Kono K, Kitahara F, lizuka H, Matsumoto Y, Fujino MA, Ooi A. Status of c-erbB-2 in gastric adenocarcinoma: a comparative study of immunohistochemistry, fluorescence in situ hybridization and enzyme-linked immuno-sorbent assay. Int J Cancer 2002; 20;98(6):833-7

10. Wang S, Zheng G, Chen L, Xiong B. Effect of HER-2/neu over-expression on prognosis in gastric cancer: a metaanalysis. Asian Pac J Cancer Prev 2011; 12: 1417-1423

11. Liu X, Xu P, Qiu H, et al. Clinical utility of HER2 assessed by immunohistochemistry in patients undergoing curative resection for gastric cancer. Onco Targets Ther 2016;9:949-58

12. Subasinghe D, Sivaganesh S, Samarsekera A, Kumarasinghe MP, Samarasekera DN, Lokuhetty MDS. Human Epidermal Growth Factor Receptor-2 in Sri Lankan Gastric Carcinoma Patients with Clinicopathological Association and Survival. Dig Dis Sci 2017;62(9):2498-2510.

13. Food and Drug Administration. In vitro companion diagnostics devices, <http://www.fda.gov/MedicalDevices/ProductsandMedicalProcedures/InVitroDiagnostics/ucm301431.htm>; 2013 [accessed 15.04.2013]. 
14. Bartley AN, Washington MK, Colasacco C, Ventura CB, Ismaila N, Benson AB 3rd, Carrato A, Gulley ML, Jain D, Kakar S, Mackay HJ, Streutker C, Tang L, Troxell M, Ajani JA.HER2 Testing and Clinical Decision Making in Gastroesophageal Adenocarcinoma: Guideline From the College of American Pathologists, American Society for Clinical Pathology, and the American Society of Clinical Oncology. J Clin Oncol 2017; 35(4):446-464.

15. Anna Sapino, Margherita Goia, Daniele Recupero, Caterina Marchiò.Current Challenges for HER2 Testing in Diagnostic Pathology: State of the Art and Controversial Issues. Front Oncology 2013; 3: 129.

16. National Cancer Control Programme Sri Lanka. Cancer Incidence Data, Sri Lanka 2001-2010. In: Ministry of Health; 2016

17. Sobin LH, Gospodarowicz M, Wittekind C. TNM Classification of Malignant Tumours. 7th ed. Wiley-Blackwell, Oxford 2009.

18. Lauren $P$. The two histological main types of gastric carcinoma: diffuse and so-called intestinal-type carcinoma. an attempt at a histo-clinical classification. Acta Pathol Microbiol Scand 1965,64:31-49

19. Josef Rüschoff, Manfred Dietel, Gustavo Baretton, Susanne Arbogast, Axel Walch, Geneviéve Monges, MariePierre Chenard, Frédérique Penault-Llorca, Iris Nagelmeier, Werner Schlake, H. Höfler, H. H. Kreipe. HER2 diagnostics in gastric cancer-guideline validation and development of standardized immunohistochemical testing. Virchows Arch. 2010 Sep; 457(3): 299-307

20. Guggenheim, D.E. and Shah, M.A. (2013) Gastric Cancer Epidemiology and Risk Factors. J Surg Oncol, 107, 230-236

21. Yang L. Incidence and mortality of gastric cancer in China. World J Gastroenterol 2006; 12: 17-20

22. Fock KM, Ang TL. Epidemiology of Helicobacter pylori infection and gastric cancer in Asia. J Gastroenterol Hepatol 2010; 25: 479-486

23. Kunz PL,Mojtahed A,Fisher GA et al. HER2 expression in gastric and gastrooesophageal junction adenocarcinoma in a US population:clinicopathological analysis with proposed approach to HER 2 assessment. Appl Immunohistochem Mol Morpho $2012 ; 20(1): 13-24$

24. Allgayer $\mathrm{H}$, Babic R, Gruetzner KU, et al. c-erb B-2 is of independent prognostic relevance in gastric cancer and is associated with the expression of tumor associated protease systems. J Clin Oncol 2000;18:2201-9.

25. De carli DM, Da rocha MP, Antunes LCM,Fagundes RB. Immunohistochemical expression of HER2 in adeno carcinoma of the stomach. Arq Gastroenterol $2015 ; 52: 152-55$

26. Ling Shan, Jianming Ying, Ning Lu. HER2 expression and relevant clinicopathological features in gastric and gastresophageal junction adenocarcinoma in a Chinese population. Diagnostic Pathology. 2013;8:76,doi 10.11.86/1746-1596-8-76.

27. James E. Boers, Harrie“tte Meeuwissen, Natalie Methorst. HER2 status in gastro-oesophageal adenocarcinomas assessed by two rabbit monoclonal antibodies (SP3 and 4B5) and two in situ hybridization methods (FISH and SISH). Histopathology 2011, 58, 383-394.

28. Hee Eun Leea, Kyoung Un Parkb, Seol Bong Yooc, Soo Kyung Namd, Do Joong Parke, Hyung-Ho Kime, Hye Seung Lee. Clinical significance of intratumoral HER2 heterogeneity in gastric cancer. European Journal of Cancer 2013;49(6):1448-1457

29. Federica Grillo, Matteo Fassan, Francesca Sarocchi, Roberto Fiocca, Luca Mastracci. HER2 heterogeneity in gastric/gastroesophageal cancers: From benchside to practice. World Journal Gastroenterology 201614 ; 22(26): 5879-5887 
30. Pyo JS, Sohn JH, Kim WH. Concordance rate between HER2 immunohistochemistry and in situ hybridization in gastric carcinoma: Systematic review and meta-analysis. International Journal Biolo Mark 2016;31(1):e110.

31. Bozzetti C, Negri FV, Lagrasta CA, Crafa P, Bassano C, Tamagnini I, Gardini G,Nizzoli R, Leonardi F, Gasparro $\mathrm{D}$, et al: Comparison of HER2 status in primary and paired metastatic sites of gastric carcinoma. Br $\mathrm{J}$ Cancer 2011, 104(9):1372-1376.

32. Kameda T, Yasui W, Yoshida $\mathrm{K}$ et al. Expression of ERBB2 in human gastric carcinomas: relationship between p185ERBB2 expression and the gene amplification. Cancer Research 1990; 50: 8002-8029.

33. Hollywood DP, Hurst HC. A novel transcription factor, OB2-1, is required for overexpression of the protooncogene c-erbB-2 in mammary tumour lines. EMBO J 1993; 12: 2369-2375.

34. Marx AH, Tharun L, Muth J, et al. HER-2 amplification is highly homogenous in gastric cancer. Human Pathology 2009; 40: 769-77.

35. Brien TP, Depowski PL, Sheehan CE, Ross JS, McKenna BJ. Prognostic factors in gastric cancer. Modern Pathology 1998; 11: 870-7.

\section{Tables}

Table 01-Comparison demographic, clinical-radiological-pathological features of SISH/HER2 positive and negative $\mathrm{GC}(\mathrm{N}=145)$

Demographic/ Clinical characteristics

HER2 positive HER 2 Negative total

Age, years

$>60$

01

79

80

06

59

65

Gender

Male

07

82

89

Female

00

56

\section{6}

Type of specimen

Endoscopic biopsy

02

74

76

Resection

05

64

69

\section{Tumour differentiation}

Well

03

35

38

Moderate

01

55

56 
Tumour location

Proximal

Distal

Primary tumor

T1

00

06

06

$\mathrm{T} 2$

00

61

61

T3

06

33

39

$\mathrm{T} 4$

01

Regional lymph nodes

(radiological/pathological)

NO

00

N1

87

Pathological malignant lymphadenopathy

(resections only)

N0

Distant metastases

(radiological)

M0

M1

66

Radiological/pathological tumor stage 
III

IV

Table 2. Concordance between the results of HER2 IHC and HER2 SISH

\begin{tabular}{lcccc}
\hline IHC score & SISH amplified & SISH non-amplified & & Concordance \\
\hline $0 \quad \begin{array}{c}(\mathrm{n}=124) \\
100 \%(124 / 124)\end{array}$ & 0 & 124 & \\
$1+\begin{array}{c}(\mathrm{n}=9) \\
100 \%(9 / 9)\end{array}$ & 0 & 9 & \\
$2+\begin{array}{c}(n=7) \\
28.5 \%(2 / 7)\end{array}$ & 2 & 5 & \\
$3+\begin{array}{c}(n=5) \\
100 \%(5 / 5)\end{array}$ & 5 & 138 & 0 & \\
\hline $\begin{array}{l}\text { Total }(n=145) \\
(140 / 145)\end{array}$ & 7 & & & $96.6 \%$ \\
\hline
\end{tabular}

Table 3: Associations of variables in the cox-regression with survival 


\begin{tabular}{|c|c|c|c|c|}
\hline Factor/ covariate & P value & $\begin{array}{c}\text { Beta } \\
\text { coefficient }\end{array}$ & $\begin{array}{l}\text { Standard } \\
\text { error }\end{array}$ & $\begin{array}{l}\text { Exponential of Beta } \\
\text { OR (CI) }\end{array}$ \\
\hline \multirow[t]{2}{*}{ SISH-positivity } & 0.001 & 2.755 & 0.846 & 15.72 (2.99 to \\
\hline & & & & $82.55)^{*}$ \\
\hline Age & $<0.001$ & 0.150 & 0.023 & $1.16(1.11 \text { to } 1.21)^{*}$ \\
\hline Gender & 0.845 & 0.050 & 0.255 & 1.05 (0.63 to 1.73$)$ \\
\hline Lauren Intestinal(I) type & 0.906 & NR & NR & NR \\
\hline $\begin{array}{l}\text { Lauren Diffuse type } \\
\text { (reference is Lauren I) }\end{array}$ & 0.989 & -0.004 & 0.299 & $0.996(0.55$ to 1.79$)$ \\
\hline $\begin{array}{l}\text { Lauren Mixed type } \\
\text { (reference is Lauren I) }\end{array}$ & 0.663 & -0.151 & 0.345 & $0.86(0.44$ to 1.69$)$ \\
\hline $\begin{array}{l}\text { Proximal location } \\
\text { (reference is distal location) }\end{array}$ & 0.341 & 0.243 & 0.255 & $1.28(0.77$ to 2.10$)$ \\
\hline TNM Stage level 1 & 0.977 & NR & NR & NR \\
\hline $\begin{array}{l}\text { TNM Staging level } 2 \\
\text { (reference is level 1) }\end{array}$ & 0.659 & 0.270 & 0.611 & $1.31(0.40$ to 4.34$)$ \\
\hline $\begin{array}{l}\text { TNM staging level } 3 \\
\text { (reference is level 1) }\end{array}$ & 0.725 & 0.225 & 0.640 & 1.25 (0.36 to 4.40$)$ \\
\hline $\begin{array}{l}\text { TNM staging level } 4 \\
\text { (reference is level } 1 \text { ) }\end{array}$ & 0.752 & 0.223 & 0.707 & 1.25 (0.31 to 4.99$)$ \\
\hline $\begin{array}{l}\text { Underwent surgery } \\
\text { (reference is biopsy for palliative } \\
\text { chemotherapy) }\end{array}$ & 0.207 & -0.687 & 0.545 & $0.50(0.17$ to 1.46$)$ \\
\hline
\end{tabular}

*statistically significant, NR-Not relevant

\section{Figures}




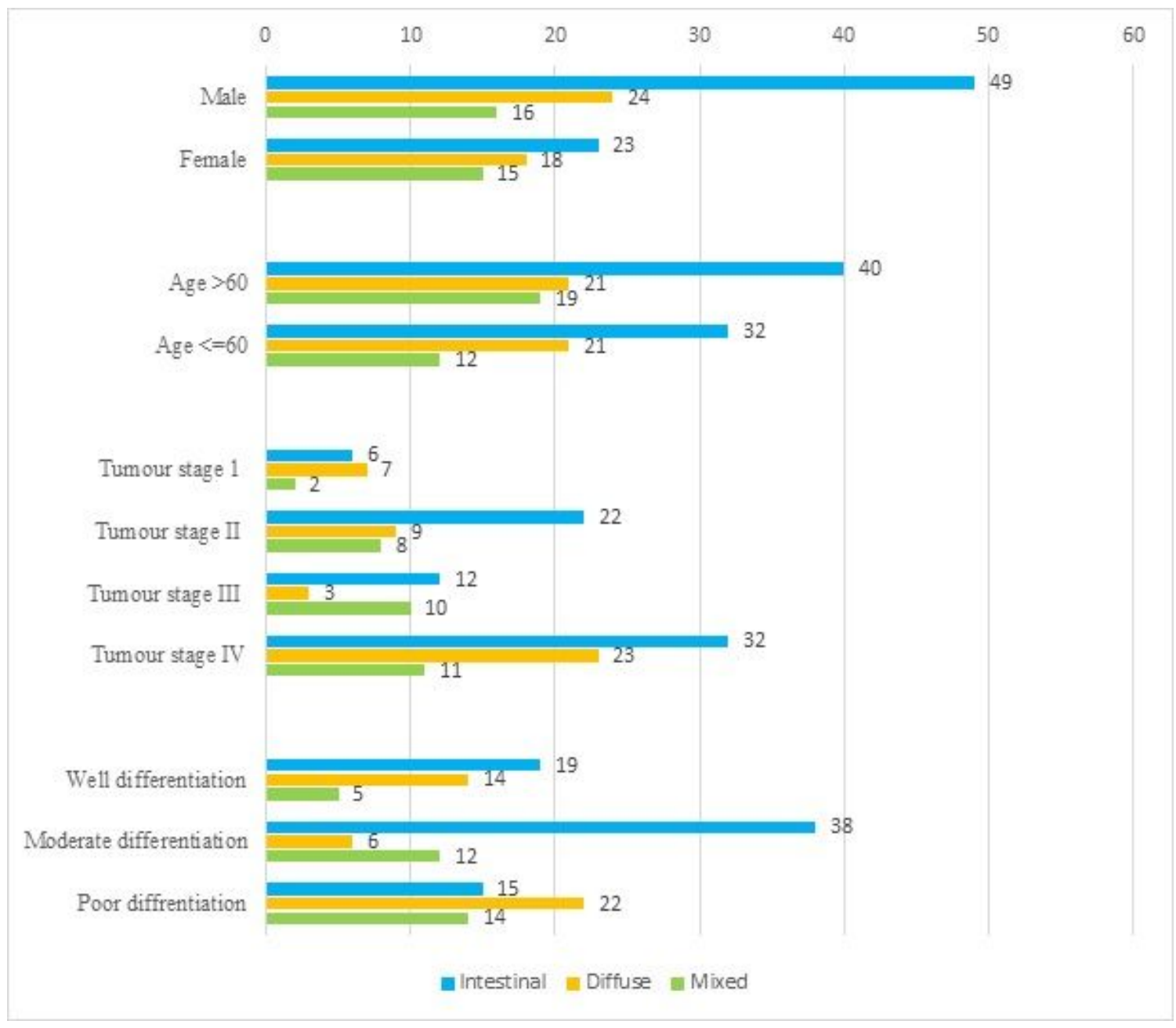

\section{Figure 1}

Histological classification and of the study population with GC when stratified under the socio-demographic and pathological characteristics* *Tumour stage (combined clinic-radiological and pathological) 


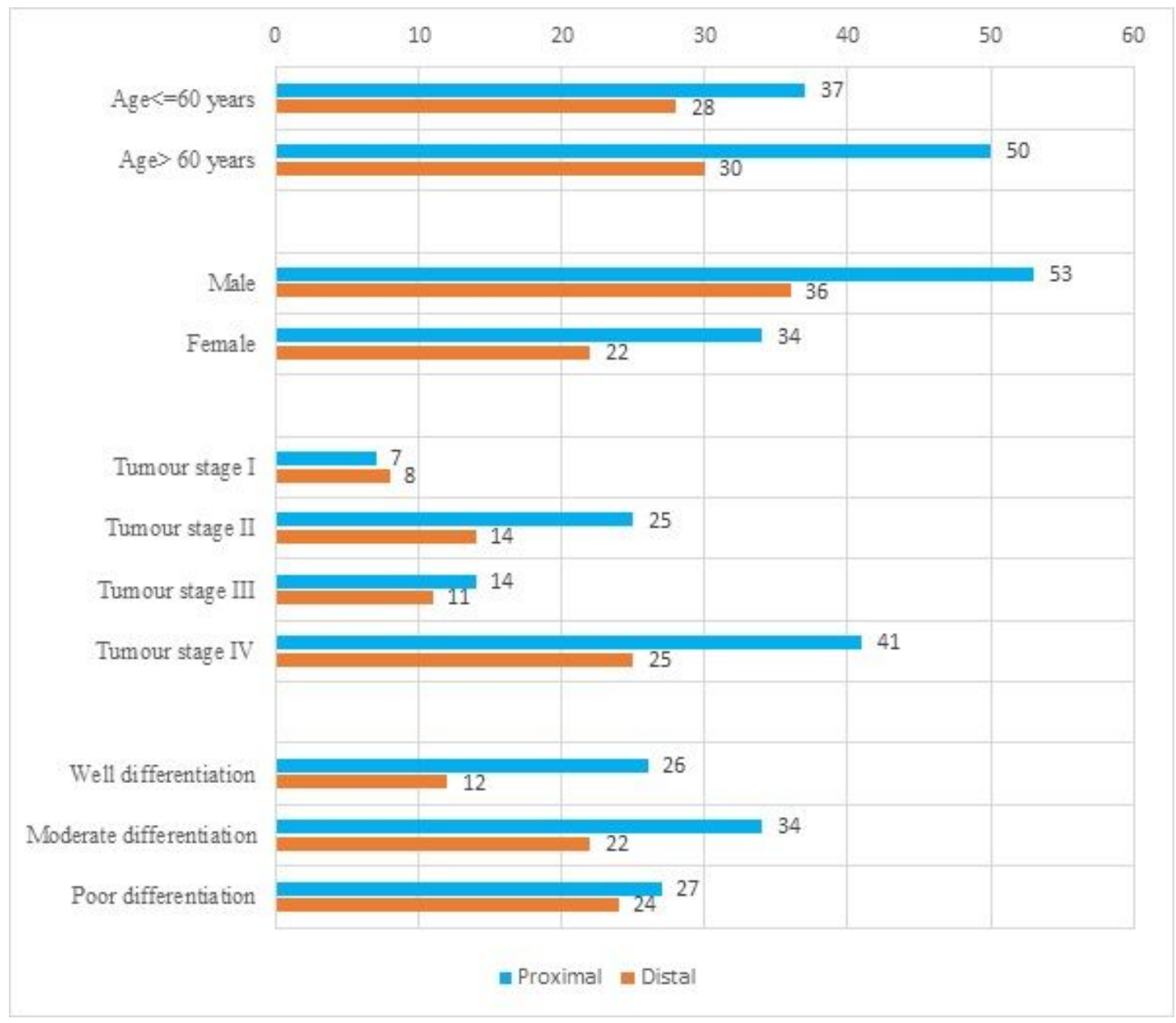

\section{Figure 2}

Tumour location of the study population when stratified under the socio-demographic and pathological characteristics * Tumour stage (Combined clinical pathological and radiological)

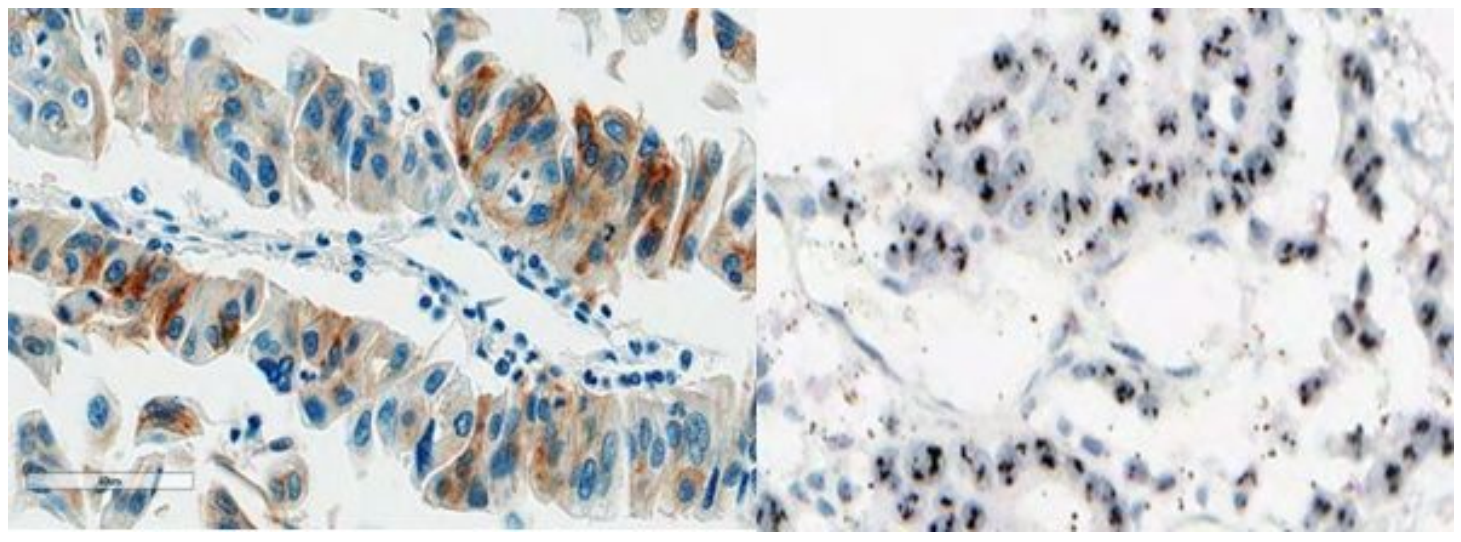

\section{Figure 3}

HER2 positive gastric carcinoma. 3A-IHC HER2 + 2 gastric carcinoma (x40) 3B-SISH amplification of IHC HER2 +2 gastric carcinoma 


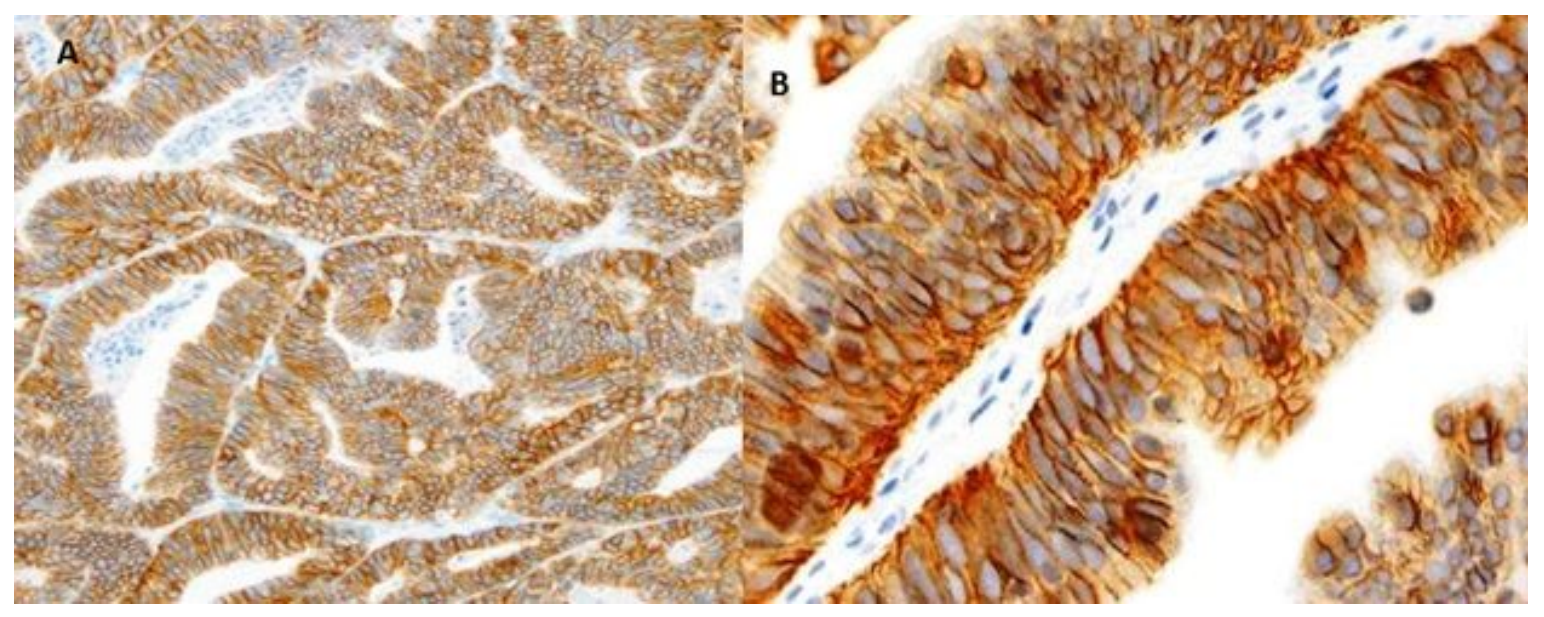

Figure 4

HER2 positive gastric carcinoma by IHC. 4A- Score 3+ tumour (20x) , 4B - Score 3+ tumour (40x)

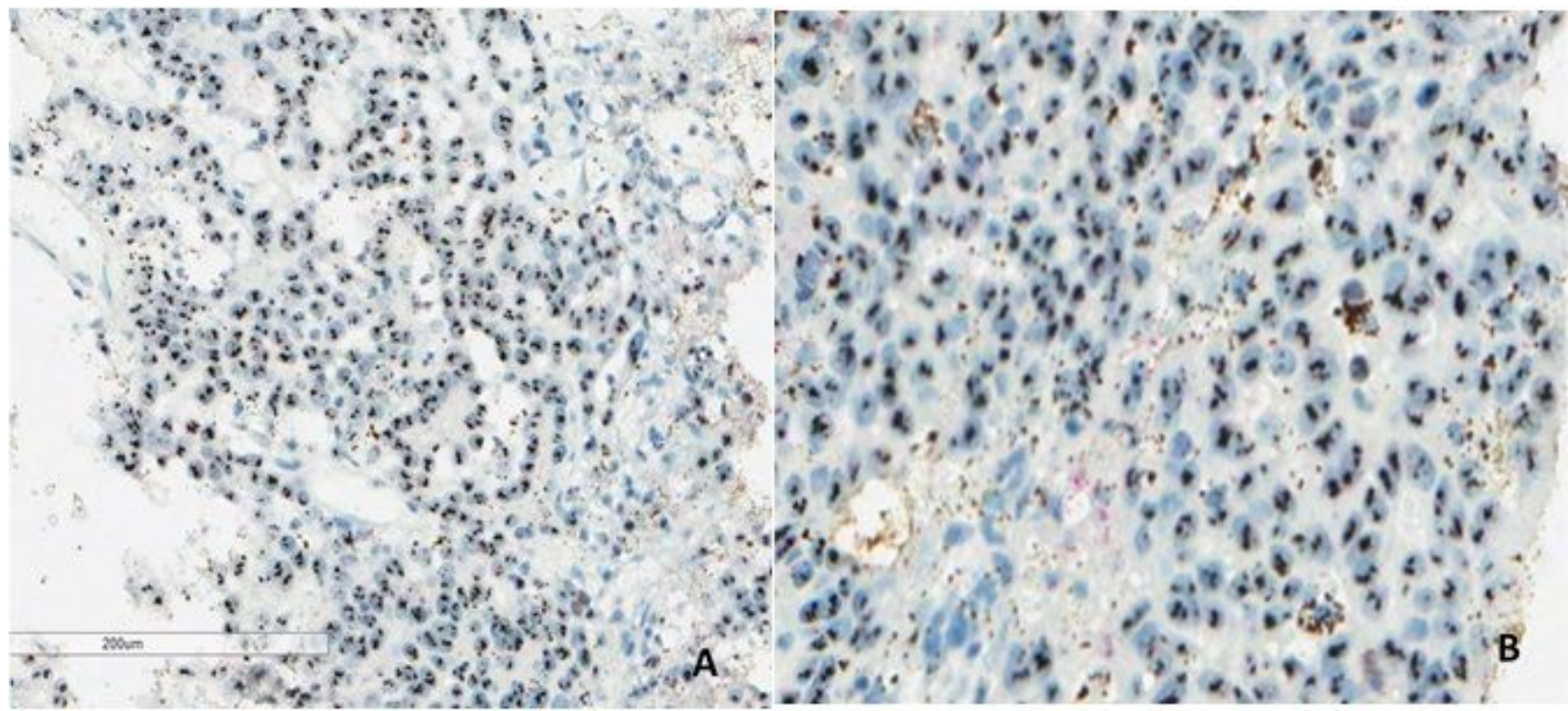

\section{Figure 5}

HER2 positive gastric carcinoma by SISH 5A-IHC +3 tumour SISH showing strong amplification with nuclear clumps (x20) 5B-IHC +3 tumour SISH showing strong amplification with nuclear clumps (x40) 


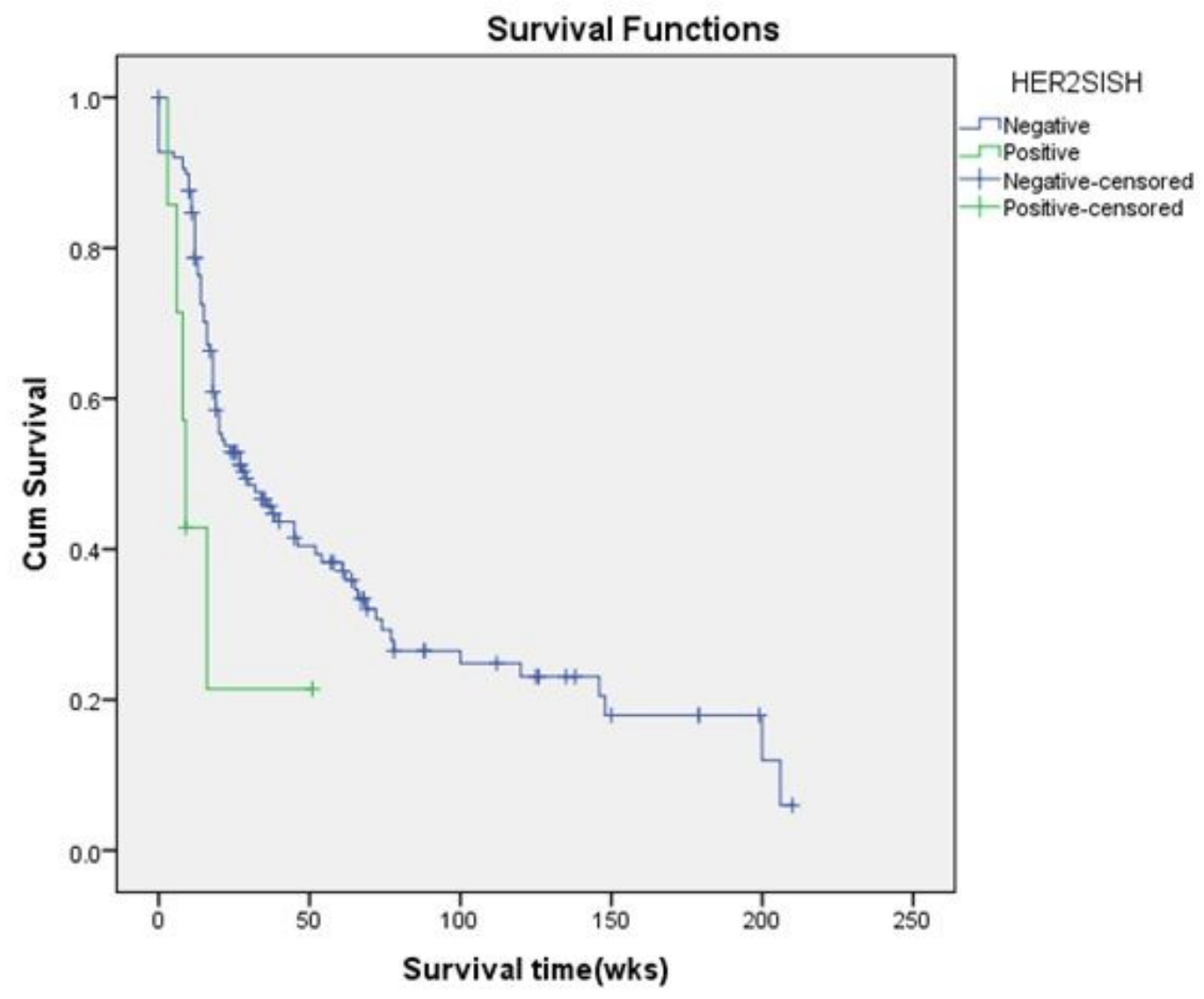

Figure 6

Kaplan-Meier survival curves for SISH HER2 positive and negative gastric carcinoma cases. Log-rank Mantel-cox test was used $(p=0.018)$ 